\title{
Study on the tuning mechanism of RF cavity for axion dark matter search experiment at IBS/CAPP in KAIST
}

\section{Youngjae Lee*}

Department of Physics, Korea Advanced Institute of Science and Technology (KAIST)

E-mail: djfldlshs@kaist.ac.kr

\begin{abstract}
About $23 \%$ of the energy density of the universe is considered to be in a form of non-baryonic dark matter. One of the strong candidates of dark matter is a hypothetical particle called the axion. In an axion dark matter search haloscope experiment, axions coherently scatter off the magneticfield potential in a frequency-tunable resonant cavity. A dynamic frequency tuning in the resonant cavity is essential to effectively scan through the relevant axion mass range. In CAPP18T axion search experiment at IBS/CAPP in KAIST, we use a rotating tuning-rod system. We develop a frequency tuning system using a Proportional Integral Derivative control and precision stepping motor system. In this presentation we report details of the tuning mechanism and accuracy of the setup which are interpreted in the axion mass parameter space.
\end{abstract}

The 39th International Conference on High Energy Physics (ICHEP2018)

4-11 July, 2018

Seoul, Korea

${ }^{*}$ Speaker. 


\section{Introduction}

Axion, which is hypothetical particle and solution of strong CP problem, is one of the strong candidates of the dark matter which is hypothetical matter and consist of roughly $23 \%$ of universe [1]. Axion haloscope is experimental method that is designed to detect the dark matter axions [2]. Detection process of the dark matter axions is inverse Primakoff effect that axions convert into real photons by interacting with virtual photons, magnetic field, in a cavity.

\section{Cavity frequency tuning}

We detect Radio Frequency (RF) signal which is essentially converted photons from the axions. Signal power of RF signal resonated by cavity and scan rate have maximum value at TM 010 mode. In our experiment, we use cylindrical cavity with $54 \mathrm{~mm}$ inner diameter and $466.6 \mathrm{~mm}$ height. TM 010 mode frequency of our cavity is $4.25 \mathrm{GHz}$. To check various mass range, we need frequency tuning system, the tuning-rod. If the tuning-rod consists of dielectric material, the TM mode frequency decreases. If using metal such as copper on the tuning-rod, the TM mode frequency increases. We rotate the tuning-rod from cavity inner wall to cavity center using a step motor. As the tuning-rod is close to cavity center, effect of the tuning-rod is strong. We use dielectric tuning-rod made of alumina and its diameter is $4.86 \mathrm{~mm}$. With this tuning-rod, we can scan from $3.68 \mathrm{GHz}$ to $4.22 \mathrm{GHz}$. Also, we prepare copper tuning-rod which can scan above $5 \mathrm{GHz}$ frequency range. Figure 1a, Figure 1b, and Figure 1c shows how frequency and scan rate are changed according to position of the tuning-rod.

We tested frequency tuning system of the cavity in the 18T HTS magnet. The results of test are shown in Figure 1d and Figure 1e. Figure 1d shows data received with NA. The lowest line in Figure $1 \mathrm{~d}$ is the TM 010 mode, and other lines are higher TM $01 \mathrm{~m}$ modes. The scan range of the frequency is from $3.71 \mathrm{GHz}$ to $4.22 \mathrm{GHz}$. Figure $1 \mathrm{e}$ is the noise spectra data received with SA. The red line is the data with the fake axion signal which is provided by the signal generator and the blue line is the data without the fake axion signal.

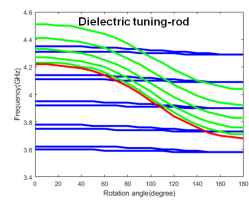

(a)

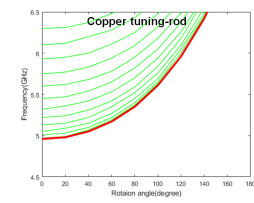

(b)

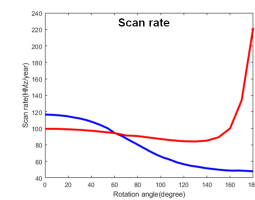

(c)

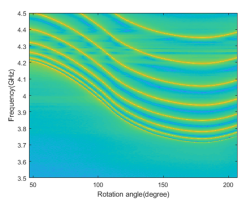

(d)

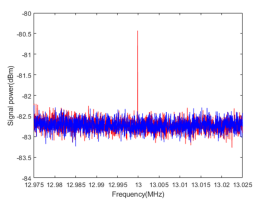

(e)

Figure 1: (a) Simulation result of dielectric tuning-rod. (b) Simulation result of copper tuning-rod (c) Expected scan rate. Blue line is when using dielectric rod, red line is when using copper rod. (d) NA result data under 18T. (e) SA result data under $18 \mathrm{~T}$

\section{References}

[1] R.D.Peccei, and H.R.Quinn, Phys. Rev. Lett. 38, 1440 (1977)

[2] P.Sikivie, Phys. Rev. B 51, 1415 (1983); L.Krauss, J.Moody, F.Wilczek, and D.E.Morris, Phys. Rev. Lett. 55, 1797 (1985) 